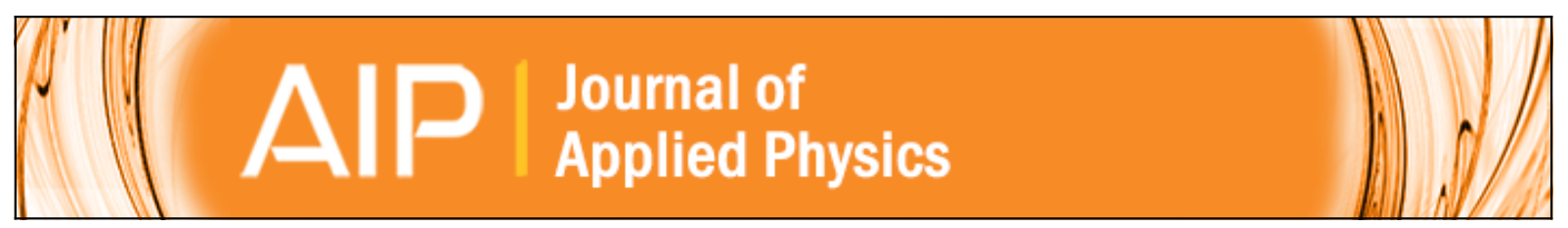

\title{
Memory effect in silicon time-gated single-photon avalanche diodes
}

A. Dalla Mora, A. Tosi, D. Contini, L. Di Sieno, G. Boso, F. Villa, and A. Pifferi

Citation: Journal of Applied Physics 117, 114501 (2015); doi: 10.1063/1.4915332

View online: http://dx.doi.org/10.1063/1.4915332

View Table of Contents: http://scitation.aip.org/content/aip/journal/jap/117/11? ver=pdfcov

Published by the AIP Publishing

\section{Articles you may be interested in}

Time-gated single-photon detection module with 110 ps transition time and up to $80 \mathrm{MHz}$ repetition rate

Rev. Sci. Instrum. 85, 083114 (2014); 10.1063/1.4893385

Afterpulse-like noise limits dynamic range in time-gated applications of thin-junction silicon single-photon avalanche diode

Appl. Phys. Lett. 100, 241111 (2012); 10.1063/1.4729389

Fully programmable single-photon detection module for InGaAs/lnP single-photon avalanche diodes with clean and sub-nanosecond gating transitions

Rev. Sci. Instrum. 83, 013104 (2012); 10.1063/1.3675579

Dark count probability and quantum efficiency of avalanche photodiodes for single-photon detection

Appl. Phys. Lett. 83, 2955 (2003); 10.1063/1.1616666

Photon generation by silicon diodes in avalanche breakdown

Appl. Phys. Lett. 73, 871 (1998); 10.1063/1.121971

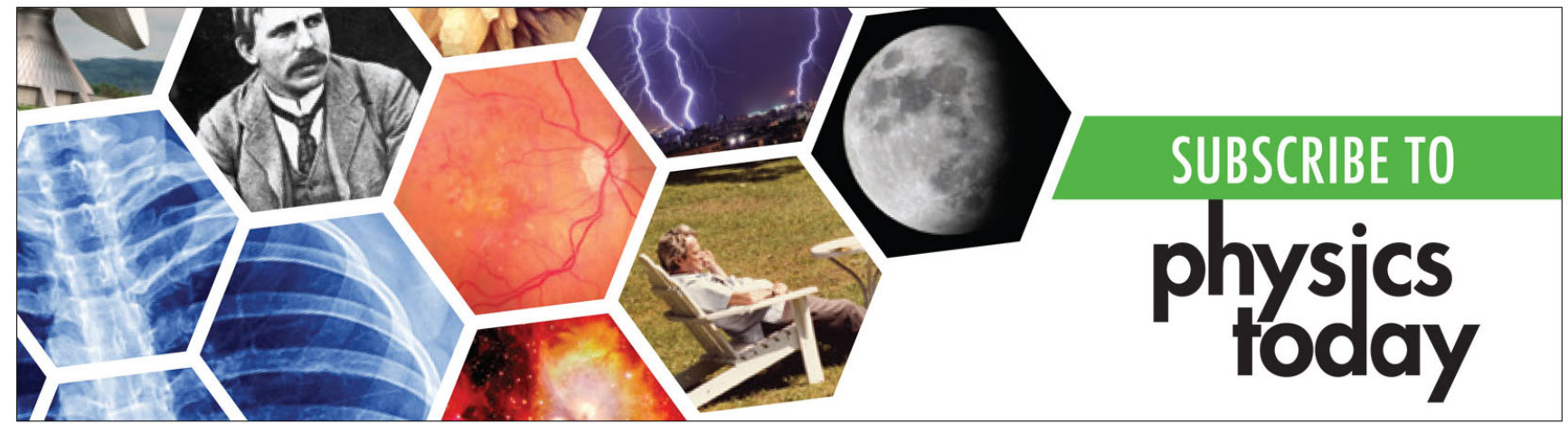




\title{
Memory effect in silicon time-gated single-photon avalanche diodes
}

\author{
A. Dalla Mora, ${ }^{1}$ A. Tosi, ${ }^{2}$ D. Contini, ${ }^{1, a)}$ L. Di Sieno, ${ }^{1}$ G. Boso, ${ }^{2}$ F. Villa, ${ }^{2}$ and A. Pifferi ${ }^{1,3}$ \\ ${ }^{1}$ Dipartimento di Fisica, Politecnico di Milano, Piazza Leonardo da Vinci 32, I-20133 Milano, Italy \\ ${ }^{2}$ Dipartimento di Elettronica, Informazione e Bioingegneria, Politecnico di Milano, Piazza Leonardo da Vinci \\ 32, I-20133 Milano, Italy \\ ${ }^{3}$ CNR, Istituto di Fotonica e Nanotecnologie, Piazza Leonardo da Vinci 32, I-20133 Milano, Italy
}

(Received 3 December 2014; accepted 7 March 2015; published online 18 March 2015)

\begin{abstract}
We present a comprehensive characterization of the memory effect arising in thin-junction silicon Single-Photon Avalanche Diodes (SPADs) when exposed to strong illumination. This partially unknown afterpulsing-like noise represents the main limiting factor when time-gated acquisitions are exploited to increase the measurement dynamic range of very fast (picosecond scale) and faint (single-photon) optical signals following a strong stray one. We report the dependences of this unwelcome signal-related noise on photon wavelength, detector temperature, and biasing conditions. Our results suggest that this so-called "memory effect" is generated in the deep regions of the detector, well below the depleted region, and its contribution on detector response is visible only when time-gated SPADs are exploited to reject a strong burst of photons. (C) 2015 AIP Publishing LLC. [http://dx.doi.org/10.1063/1.4915332]
\end{abstract}

\section{INTRODUCTION}

Emerging applications in photonics requiring singlephoton counting and accurate time-of-flight measurement demand extreme detection performances. A clear example is the case of recent advances on time-resolved diffuse optical imaging in turbid media, where the distance between illumination and collection points must be reduced so as to increase image contrast, spatial resolution, and number of collected photons at any arrival time. ${ }^{1-5}$ In these experiments, fast timegated photon detection is mandatory to reject the huge amount of "early" scarcely diffused photons (reflected by the superficial outer layers of the medium under investigation), while collecting only the "late" deep-travelling photons many decades lower (carrying useful information on deep tissues, matter, organs). Similar requirements on dynamic range arise, for example, also from fluorescence lifetime microscopy, ${ }^{6,7}$ molecular imaging, ${ }^{8}$ ultra-fast time-of-flight imaging, ${ }^{9}$ and quantum information. ${ }^{10}$

To address these applications, we have recently developed a time-gated detection module based on thin-junction Single-Photon Avalanche Diode (SPAD) ${ }^{11}$ and the related measurement technique ${ }^{12}$ able to increase the dynamic range of time-resolved optical measurements up to 8 orders of magnitude (from the main "early" photon peak down to the fainter "late" photon signal), thus allowing to investigate biological media down to few centimeters. On the one hand, this result opened the way to perform time-resolved optical measurements in extreme situations (e.g., when the useful signal is overwhelmed by a much larger amount of leading unwelcome photons). On the other hand, it highlighted an unknown source of background noise in thin-junction silicon SPADs, due to a sort of "memory effect" (ME), whose discovery and preliminary characterization we reported in

\footnotetext{
a) Author to whom correspondence should be addressed. Electronic mail: davide.contini@polimi.it
}

Ref. 13. This effect causes the noise avalanche ignitions to be not uniformly distributed within the time-gated window, thus resulting in a background therein that is decaying instead of being flat.

The SPAD background noise is usually given by the sum of two contributions: (i) dark count rate (DCR) due to both temperature-assisted processes (thermal generation of free carriers, dominated by the Shockley-Read-Hall mechanism within the depleted region) ${ }^{14}$ and field-assisted processes (direct band-to-band tunneling and trap-assisted tunneling); (ii) afterpulsing, due to carriers trapped within the depleted region during a primary avalanche multiplication process and later released, thus triggering again the detector. ${ }^{15}$

Some other noise sources have been recently characterized. In thick-junction silicon SPADs, a "twilight effect" similar to ME was noticed. ${ }^{16,17}$ However, thick- and thinjunction SPADs are quite different in both geometry and electric field profile. Moreover, twilight phenomenon exhausts in few nanoseconds from the excitation light pulse, while ME lasts few microseconds, hence it has different physical origins.

A phenomenon named charge persistence (or subsistence) was observed in InGaAs/InP SPADs, mainly when employed in optical time-domain reflectometry. ${ }^{18-20}$ To the best of our knowledge, the physical process generating such charge persistence is not clear, but Eraerds et al. ${ }^{21}$ ascribed it to the filling of the same trapping centers that give rise to afterpulsing. Indeed, when photons hit the detector's active area during the OFF state (i.e., when the reverse bias voltage is below breakdown), photo-generated electron-hole pairs can still impact ionize hence be multiplied due to the residual finite gain of the detector. This process is similar to what happens in linear-mode avalanche photodiodes. Instead ME in silicon SPADs cannot be ascribed to a similar kind of trapping since the decaying time constants are much longer than those of afterpulsing and they do not depend on the electric field, ${ }^{13}$ as it might 


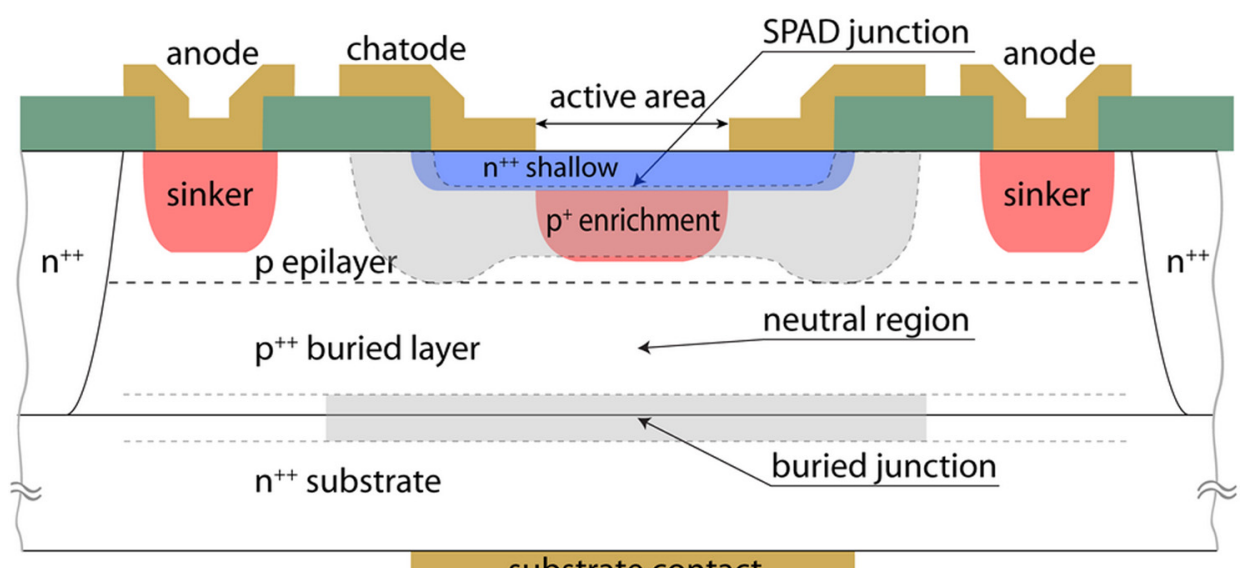

FIG. 1. Sketch (not in scale) of the characterized thin-junction customtechnology single-photon avalanche diode.

be expected in Poole-Frenkel and carrier-phonon coupling effects. $^{22}$

Very recently, Lunghi et al. ${ }^{23}$ reported the characterization of a charge persistence phenomenon in both thick- and thin-junction silicon SPADs. The process they observed is different from ME since: (i) the decay time constants clearly change with temperature, thus suggesting a trapping mechanism; (ii) the decay time constant is independent of the light wavelength. Vice versa, in this paper we show a different behavior of $\mathrm{ME}$ by changing either the detector temperature or the photon wavelength.

Therefore, memory effect, charge persistence, and twilight effect can give rise to an afterpulse-like noise in optical measurements with SPADs, but the dominant one can differ, depending on detector kind, geometry, and operating conditions.

From our first discovery of the memory effect in a custom-technology thin-junction silicon SPAD (whose structure is sketched in Fig. 1), we demonstrated: ${ }^{13}$ (i) ME is generated when the detector is exposed to a strong illumination and linearly increases with the number of incoming photons; (ii) ME decays with time constants longer than those ascribed to classical afterpulsing processes in silicon SPADs; (iii) ME has decay time constants not sensitive to the electric field applied to the junction; (iv) differently from classical afterpulsing, ME arises even if the detector is kept OFF during the illumination phase, by lowering the reverse voltage below breakdown (thus quite inhibiting any charge carrier multiplication within the depletion region). Considering all these findings, we speculated that $\mathrm{ME}$ is not due to carrier trapping within the depleted region, but it can arise from a physical process occurring outside it, where the electric field is negligible.

In this paper, we present a comprehensive experimental characterization of the memory effect and we propose a possible phenomenological understanding.

\section{MATERIALS AND METHODS}

Fig. 2(a) shows the Time-Correlated Single-Photon Counting (TCSPC) setup we employed for ME characterization, while Fig. 2(b) shows the detector front-end circuitry for SPAD biasing, gating, and signal read-out (the parasitic $\mathrm{p}-\mathrm{n}$ diode between anode and substrate is also highlighted).
The pulse generator tasks are: (i) to provide double or single gate window to the detector at a repetition rate of $10 \mathrm{kHz}$; (ii) to trigger both the laser pulse excitation and the time-toamplitude conversion of a multichannel analyzer (Varro 16k, Silena, Italy); (iii) to provide a reset pulse to restore the SPAD operating conditions just after each gating pulse. The pulsed laser control unit (PDL-800, Picoquant $\mathrm{GmbH}$, Germany) is used together with different laser heads in order to investigate different excitation wavelengths. A variable attenuator is used to adjust the number of photons on the detector. The gated SPAD and its ancillary electronics (extensively described in Ref. 2) are placed into a climatic chamber, in order to characterize the ME dependence on temperature. The avalanche pulse, due to either photon absorption or noise, sets the conversion stop signal to the multichannel analyzer.

As depicted in Fig. 2(c), the strong laser pulse (well above the single-photon level) hits the SPAD just before the end of the first gate window (whose time duration is $\mathrm{T}_{\mathrm{ON}, 1}=100 \mathrm{~ns}$ ),
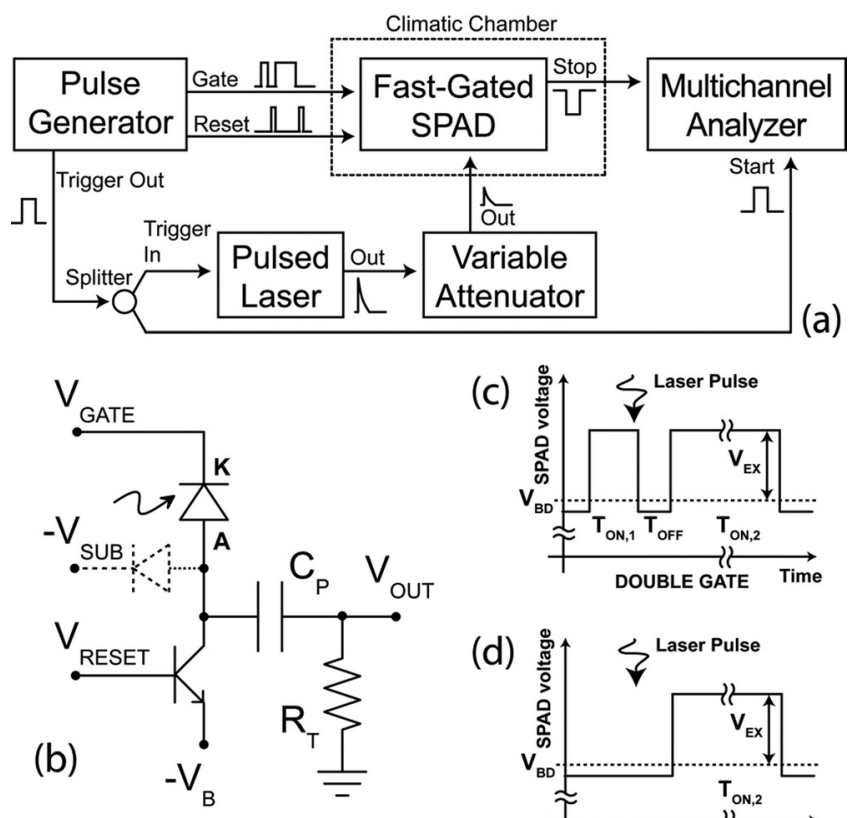

(c)

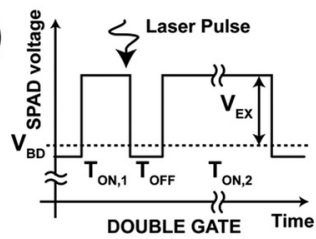

(d)

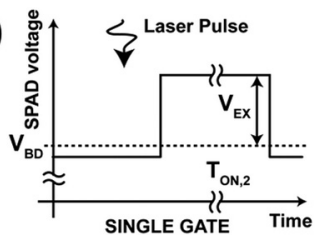

FIG. 2. (a) Instrumental setup; (b) simplified schematic of SPAD front-end circuitry; (c) double and (d) single gating schemes. 
thus triggering an avalanche with almost $100 \%$ probability. After the following OFF-time $\left(\mathrm{T}_{\mathrm{OFF}}=45 \mathrm{~ns}\right.$ ), a second gate window (whose time duration is $\mathrm{T}_{\mathrm{ON}, 2}=5 \mu \mathrm{s}$ ) is opened, when the laser is OFF, hence only afterpulsing and other dark count generation phenomena could trigger an avalanche and be detected.

The pulse generator allows also a second working mode, shown in Fig. 2(d): the first gating window is removed (i.e., the detector is biased $0.5 \mathrm{~V}$ below breakdown), in this way photo-generated carriers experience just a finite (not nil) gain, hence the avalanche cannot self-sustain as in Fig. 2(c). In the followings, we will refer to these two working modes as Double Gate Configuration (DGC) and Single Gate Configuration (SGC), respectively.

In Ref. 13, we demonstrated that the difference in background decays collected within the second window by switching between the DGC and the SGC is only due to the afterpulsing contribution, which is dominant only in the DGC. Indeed, in the presence of the first gate window, the avalanche can self-sustain and so the number of carriers within the junction is orders of magnitude higher than in SGC, thus boosting the trapping process. Vice versa, since ME is supposed not to be generated from the depleted region, switching between DGC and SGC (i.e., changing the electric field during the illumination phase) is expected not to impact ME.

The detectors under investigation are customtechnology thin-junction SPADs from Politecnico di Milano, whose characteristics (device layout and performance) are described in Refs. 24 and 25. The attenuator is set to reach a photon count rate of few thousands counts per second within the second gating window in order to speed up the measurements. Hence, all recorded data (5000 s integration time) are corrected for the pile-up distortion following the algorithm by Coates $^{26}$ and the constant primary dark count rate is then subtracted.

\section{MEASUREMENTS AND DISCUSSION}

Fig. 3 shows multi-exponential decays acquired during the second gate window when a $100 \mu \mathrm{m}$ active area diameter SPAD is saturated by a light pulse at $973 \mathrm{~nm}$ during the first gate window and the climatic chamber is set to $0{ }^{\circ} \mathrm{C}$. Curves were acquired in both DGC and SGC configurations, at two different excess bias $\left(\mathrm{V}_{\mathrm{EX}}\right.$, i.e., the difference between the reverse bias voltage and the breakdown voltage), while the mean power of the impinging laser was constant (about $5 \mu \mathrm{W})$ and the substrate node $\left(\mathrm{V}_{\mathrm{SUB}}\right.$ in Fig. 2(b)) was left floating. The computed difference between curves acquired in DGC (solid lines) and SGC (dotted lines), due to the afterpulsing process during the DGC illumination phase, is also shown in Fig. 3 (dashed lines). It is worth noting that in DGC afterpulsing is dominant only in the early part of the background decay (below $0.5 \mu \mathrm{s}$ ), thus proving that it is described by faster time constants, which are definitely absent in SGC instead. Moreover, as expected by the Poole-Frenkel effect and the carrier-phonon coupling theory, the higher the electric field, the higher the trapped carrier release rate, as confirmed by the computed curves. For this

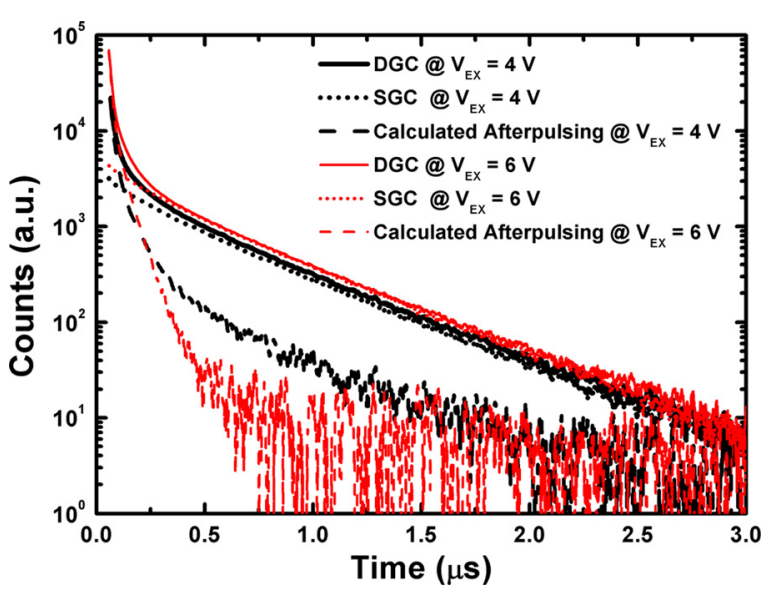

FIG. 3. Examples of decays during the second gate window in double gate configuration (DGC, solid line) and single gate configuration (SGC, dotted line) at two excess bias voltages, at the same laser power, and at $0{ }^{\circ} \mathrm{C}$. The dashed lines show the calculated afterpulsing decays.

reason, when SPADs are employed with only one time-gated window (as in SGC), e.g., to avoid detecting "early" photons, the memory effect is indeed the dominant source of noise, since the afterpulsing induced by early photons is negligible.

In order to characterize the absolute ME intensity at different wavelengths, we acquired curves in SGC by keeping the laser photon rate constant while changing the light wavelength. In this way, by integrating the total number of counts (after pile-up correction and primary dark counts subtraction) and dividing by the total number of photons reaching the device, it is possible to obtain the memory effect triggering efficiency. Fig. 4 shows a superimposition of the SPAD photon detection efficiency at $\mathrm{V}_{\mathrm{EX}}=5 \mathrm{~V}$ and the ME triggering probability at room temperature, on two different vertical scales for proper comparison. The high ME efficiency region is shifted towards longer wavelengths with respect to the photon detection efficiency spectrum, thus implying that the phenomenon is triggered in deeper regions of the detector, well below the depleted region, where longer wavelength photons are absorbed. We verified that the increase in ME amplitude by raising excess bias is in good agreement with the increased avalanche triggering probability in the depleted region (data not shown), where the avalanche (triggered

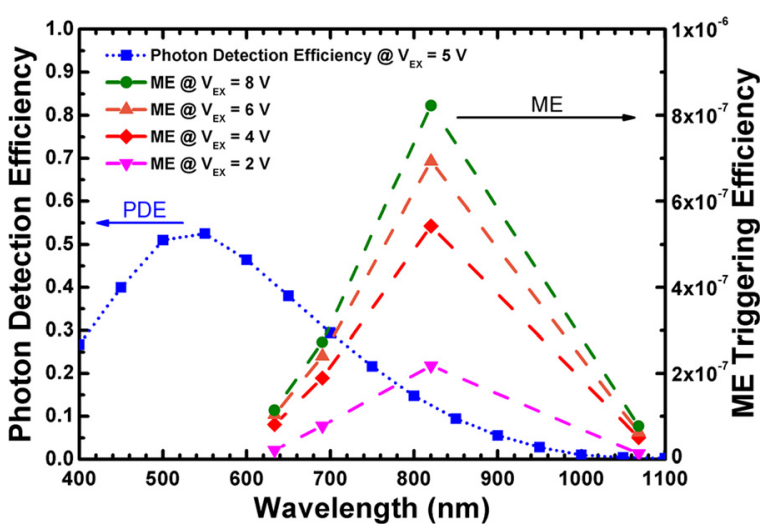

FIG. 4. Comparison between SPAD photon detection efficiency and memory effect triggering efficiency at different excess bias voltages. 
either by photons, dark generated carrier, afterpulsing, or ME) takes place.

The ME temporal decay can be well fitted with the sum of two exponentials. Fig. 5 shows the fitted parameters at the same wavelengths of Fig. 4 and at different excess bias voltages. By computing the ratio between the amplitude $\left(A_{l}\right)$ of the fast time constant $\left(\tau_{1}\right)$ and the amplitude $\left(A_{2}\right)$ of the slow one $\left(\tau_{2}\right)$, it is possible to appreciate a progressive reduction of the fast term by increasing the wavelength (at $1068 \mathrm{~nm}$ the fast term is negligible). A possible explanation of this phenomenon is that $\mathrm{ME}$ is generated in two distinct areas of the detector, at different depths below the depleted region, thus showing two different exponential decays.

We also characterized the dependence of ME on detector temperature in $\mathrm{SGC}$, between $-30{ }^{\circ} \mathrm{C}$ and $+30^{\circ} \mathrm{C}$ at $15^{\circ} \mathrm{C}$ steps. Fig. 6 shows the measurements at two excess bias $(4 \mathrm{~V}$ and $6 \mathrm{~V})$ and at two wavelengths $(690 \mathrm{~nm}$ and $973 \mathrm{~nm}$ ). It is clear that the higher the temperature, the higher the memory effect amplitude, but the time constants of the bi-exponential (at $690 \mathrm{~nm}$ ) or single-exponential (at $973 \mathrm{~nm}$ ) decays are not sensitive to detector temperature. Such ME temperature independence is not compatible with a carrier trapping phenomena since: (i) the decay time constants should decrease as the temperature increases, similarly to afterpulsing effect shown in Fig. 3, according to a carrierphonon coupling process; ${ }^{15}$ (ii) the amplitude should decrease as the temperature increases due to the increased release rate of carrier trapped during the previous OFF-time; (iii) in a trapping phenomenon, the independence of the time

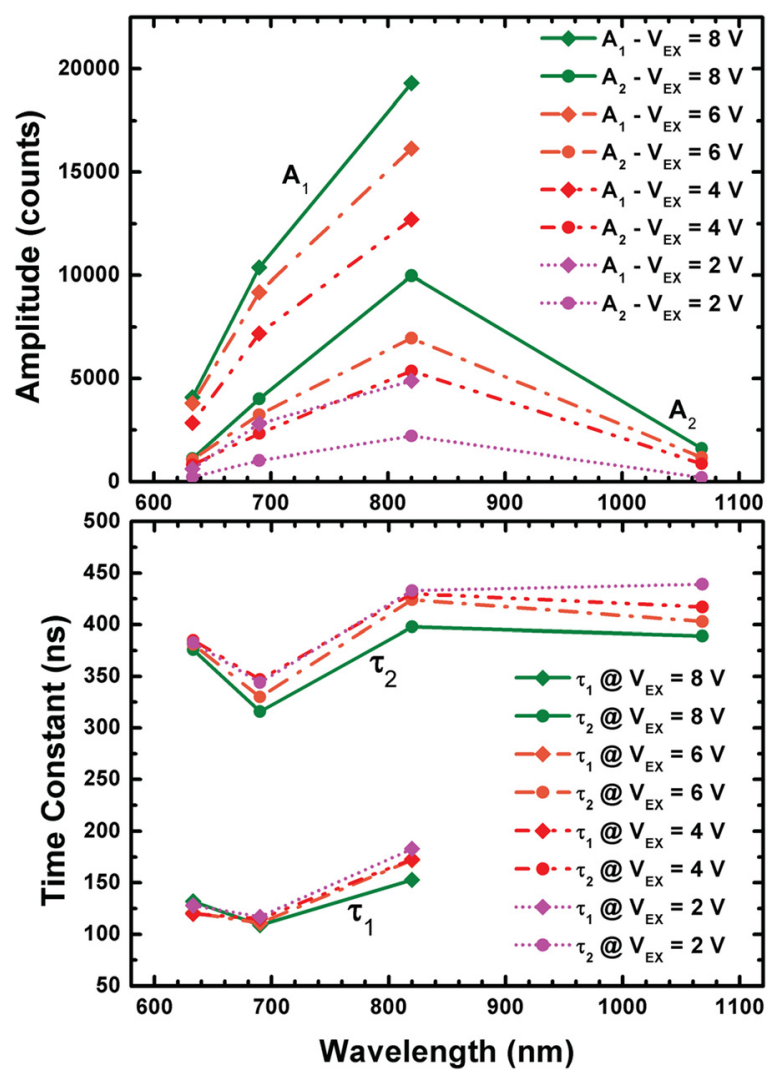

FIG. 5. Amplitude of the different exponential components of the ME decay (top) and their time constants (bottom) at different wavelengths. constant on temperature would imply the presence of trapping states having a quasi-null activation energy, but this hypothesis can be discarded because of the long time constants measured, which are expected to become much shorter in case of shallow traps within the detector depleted region.

A possible explanation of the ME origin is a slow diffusion of carriers photogenerated in the deepest layers of the device. This hypothesis is in agreement with the low temperature dependence of the diffusion coefficient $D_{n}$ of electrons in the considered temperature range $\left(D_{n} \sim 20 \mathrm{~cm}^{2} / \mathrm{s}\right.$, with less than $10 \%$ variation between $-30^{\circ} \mathrm{C}$ and $\left.+30^{\circ} \mathrm{C}\right)^{14,27}$ and with the high doping concentration level of the detector buried layer and substrate (in the order of $10^{17} \mathrm{~cm}^{-3}$ ). ${ }^{28}$

In order to verify possible effects due to the presence of the secondary depleted region beneath the buried layer (see the $\mathrm{p}^{++} / \mathrm{n}^{++}$buried junction in Fig. 1), we performed background decay measurements at constant laser power at $672 \mathrm{~nm}$, while biasing the SPAD substrate $\left(\mathrm{V}_{\mathrm{SUB}}\right)$ at different voltages. The idea was to modify the possible carrier diffusion from the substrate towards the main depleted region. At $\mathrm{V}_{\text {SUB }}$ close to the anode voltage $\left(\mathrm{V}_{\mathrm{A}}\right.$, i.e., $27.5 \mathrm{~V}$ during the detector quiescence, at room temperature), the parasitic substrate diode is close to the forward bias $\left(\mathrm{V}_{\mathrm{A}-\mathrm{SUB}}\right.$ close to $0 \mathrm{~V}$, see Fig. 1) and measurement cannot be performed because of the high probability of entering into a latch-up condition, due to the positive anode voltage transient when an avalanche is triggered. ${ }^{29}$ Vice versa, in the range between $22 \mathrm{~V}$ and $6.2 \mathrm{~V}$ (i.e., $\mathrm{V}_{\mathrm{A}-\mathrm{SUB}}$ from $5.5 \mathrm{~V}$ to $21.3 \mathrm{~V}$ of reverse bias) no background decay can be detected during the second gate window, so no memory effect is present or it is strongly suppressed. By further increasing the substrate voltage from $6 \mathrm{~V}$ to $5 \mathrm{~V}$ at $0.2 \mathrm{~V}$ step, a strong increase in the background decay can be noticed, as shown in Fig. 7. Finally, voltages higher than $-5 \mathrm{~V}$ (i.e., $\mathrm{V}_{\mathrm{A}-\mathrm{SuB}}$ higher than $21.5 \mathrm{~V}$ of reverse bias) are again forbidden due to a steep increase in the detector count rate due to the substrate breakdown occurring because of the high reverse bias applied to that $\mathrm{p}^{++} / \mathrm{n}^{++}$ junction (whose breakdown voltage is $22.2 \mathrm{~V}$ at room temperature).

From measurements reported in Fig. 7, it is possible to conclude that the noise is generated below the buried layer since both the amplitude and decay time constant are strongly dependent on the reverse bias applied to the substrate junction. It is worth noting that the background noise decay acquired in Fig. 7 is probably due to a different physical process with respect to the one measured by leaving the substrate floating (e.g., the phenomenon reported in Fig. 6): in the former case, the secondary depleted region is strongly reverse biased, close to the breakdown level, while in the latter it is free to change its voltage around the built-in condition, thus resulting in a very low reverse voltage. Hence, the reason why carriers photogenerated within the substrate can diffuse towards the multiplication region is probably different. However, we verified that the background decay shapes acquired by biasing the buried junction close to its breakdown level (Fig. 7) is similar to those acquired by leaving the substrate floating (Fig. 6), thus suggesting that in both cases the phenomenon is triggered by photogenerated 

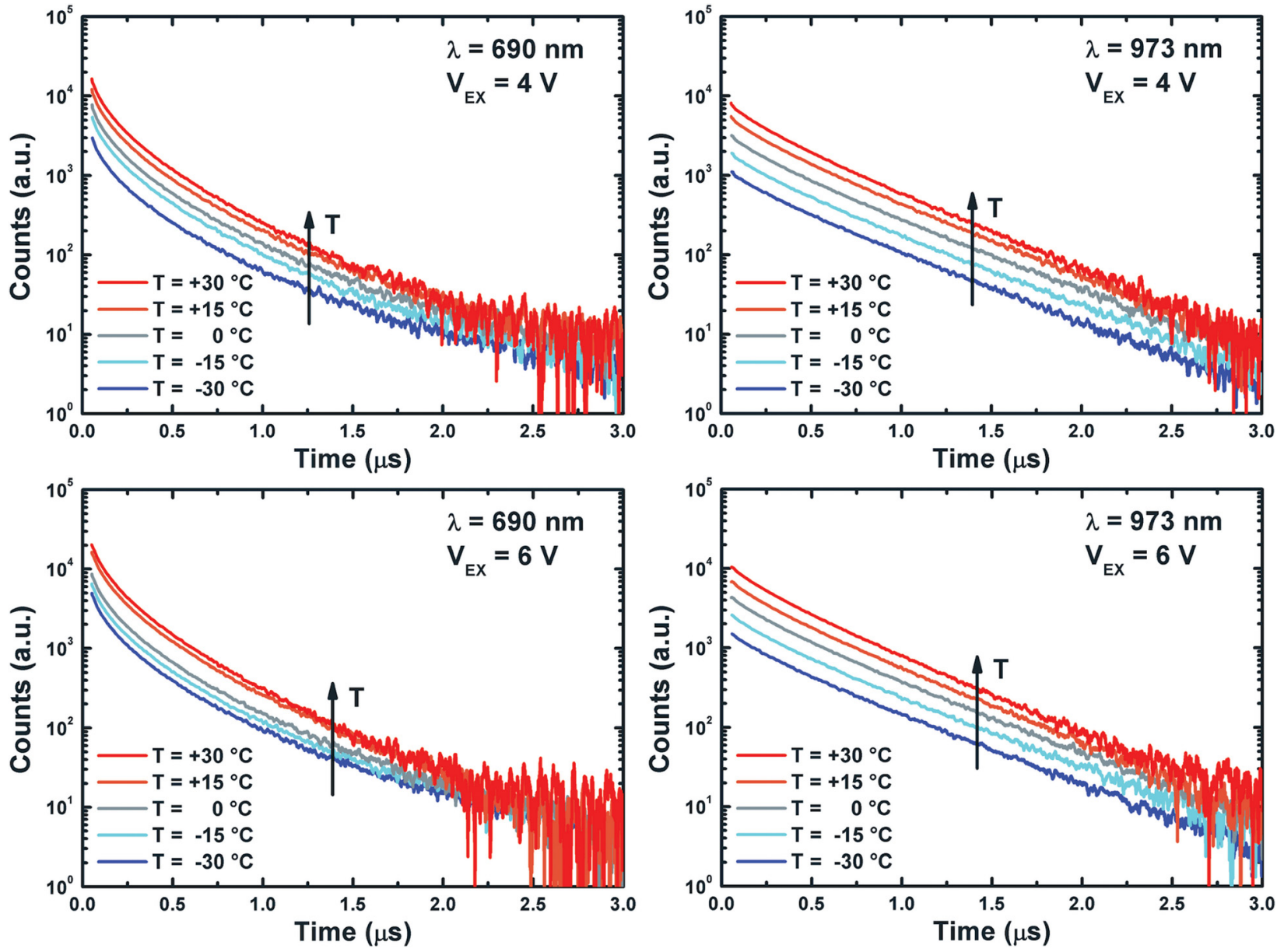

FIG. 6. Background decays acquired in single-gate configuration (SGC) at different temperatures, wavelengths (left and right), and excess bias (top and bottom).

electrons diffusing towards the SPAD junction from the detector substrate.

In order to verify the effective ME suppression at $\mathrm{V}_{\mathrm{SUB}}$ voltages between $22 \mathrm{~V}$ and $6.2 \mathrm{~V}$ in the same biasing configuration of Fig. 7, we increased the incoming laser power in SGC. Fig. 8 represents the comparison between a DGC measurement (dotted line) acquired at the same laser power of Fig. 7 and an SGC one (solid line) acquired with the laser

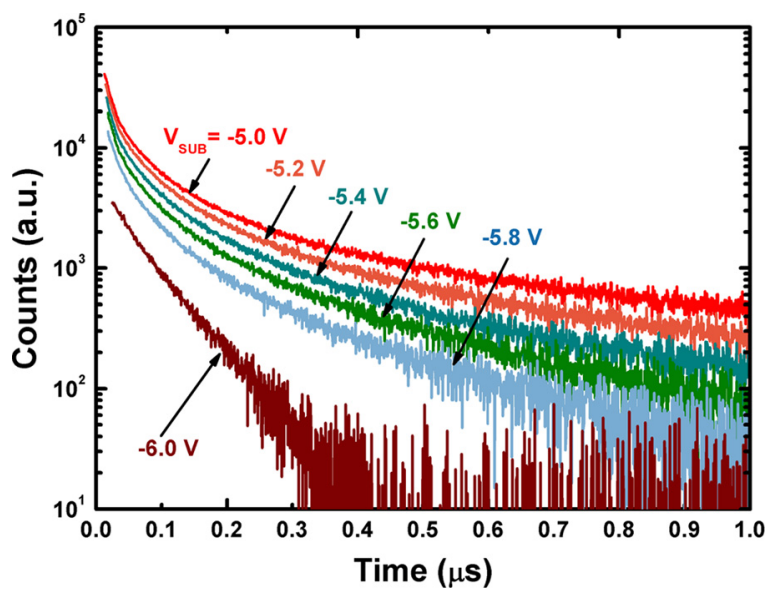

FIG. 7. Background decays within the second gate window, acquired in single-gate configuration (SGC) at different substrate bias voltages, at $25^{\circ} \mathrm{C}$. power increased by a factor of $5 \times 10^{4}$ (by tuning the variable optical attenuator), at $\mathrm{V}_{\mathrm{SUB}}=-10 \mathrm{~V}$ (i.e., $\mathrm{V}_{\mathrm{A}}$ $\mathrm{suB}=-17.5 \mathrm{~V}$ of reverse bias). This measurement shows that the ME is still present, but with a triggering efficiency 4 orders of magnitude lower with respect to the floating substrate condition, since a 4 orders of magnitude higher optical power is needed to detect a background decay (with an

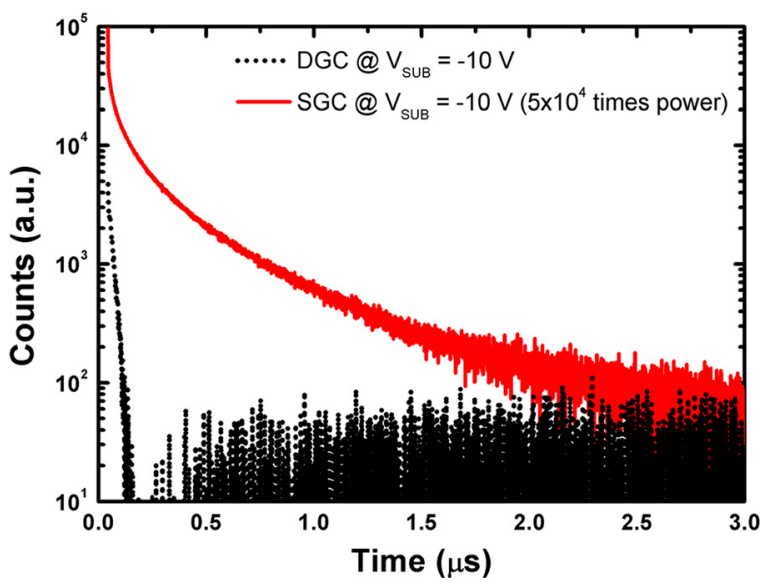

FIG. 8. Background decays during the second gate window with (DGC, dots) and without (SGC, solid line) the first gate window. In SGC, the laser pulse in the first gate window was increased by a factor of $5 \times 10^{4}$ compared to DGC. 
integral of the same order of magnitude shown in Fig. 7) using the SGC. Hence, a further extension of the dynamic range when using fast-gated SPADs from 8 (demonstrated in Ref. 12 without biasing the detector substrate) to 12 orders of magnitude is feasible by properly biasing the detector substrate.

The physical process involved in ME generation is still not fully clear and further investigations are needed to provide a complete explanation. The increase in the amplitude of ME by increasing temperature (see Fig. 6) could be only partially explained by considering the dependence of silicon absorption coefficient on temperature. ${ }^{14}$ Indeed, in the range between $-30{ }^{\circ} \mathrm{C}$ and $+30^{\circ} \mathrm{C}$, the energy gap of (intrinsic) silicon is expected to change between $1.132 \mathrm{eV}$ and $1.115 \mathrm{eV},{ }^{30}$ thus also increasing the number of photogenerated electron-hole pairs in the detector below the depleted region. We already reported similar energy band-gap narrowing effects with temperature in thin-junction silicon SPAD when using longer wavelengths. ${ }^{31}$ However, this small variation of the energy band-gap is expected to impact the number of photogenerated carriers much less (lower than $10 \%)^{32}$ than one order of magnitude as reported in Fig. 6 , when changing the device temperature by $60^{\circ} \mathrm{C}$; therefore, the dominant effect should have a different explanation.

The thin-junction SPADs under test have a double epitaxy structure, which was introduced to limit the number of electrons that, generated in the deepest layers of the device (substrate), can reach the depleted region with an excessive delay, thus giving birth to a long "diffusion" tail in the time response. ${ }^{28}$ A reverse biased substrate junction can prevent the high transit of electrons coming from deeper levels, but probably the suppression ratio is finite and affected by the substrate bias. Moreover, the presence of the highly p-doped buried layer should further reduce the possibility that an electron can diffuse towards the detector junction, due to the increased recombination rate at high doping level. However, the SPAD devices under test have a very thin buried layer (about $1.5 \mu \mathrm{m}$ ), thus possibly allowing the diffusion of a small number of electrons from the bottom. Considering the number of photons impinging onto the detector when measuring the ME (in the order of $\sim 10^{7}-10^{12}$ ), one should consider the possibility that few electrons, photogenerated within the substrate, can cross both the junction and the buried-layer, thus possibly reaching the multiplication junction and succeeding in triggering an avalanche therein. Additionally, when the substrate is left floating, the junction voltage is only given by the built-in voltage; therefore, the substrate voltage can be easily modified by the charge produced during the strong light pulse, thus moving the junction towards the forward bias condition. In this case, more electrons coming from the $\mathrm{n}^{++}$doped substrate are able to cross the junction and then be injected into the neutral region below the main SPAD depleted junction, thus possibly reaching it before recombining. Therefore, the strong dependence on the temperature of the memory effect could be ascribed to the exponential dependence of junction forward current with temperature. ${ }^{14}$

Still, it is worth noting that measurements in Fig. 7 report a different phenomenon. In that case, the substrate junction is strongly reverse biased, even approaching the breakdown level. As shown, the higher the reverse voltage, the higher the memory effect. Even if the reverse bias of the substrate junction should ensure that no electrons can reach the SPAD junction, Ref. 29 highlighted a "trap-less afterpulsing" mechanism, by simulations of a CMOS SPAD, in which charge carriers can flow in the opposite direction with respect to the main carriers flow of the avalanche current. Such phenomenon is supposed to be due to the suppression of the depleted region occurring during junction breakdown because of the large amount of flowing carriers. Hence, during the breakdown of the anode-substrate junction, some electrons could again leave the substrate, cross the buried junction, reach the SPAD junction, and trigger the detector, thus giving rise to the memory effect. Indeed, as shown in Fig. 7, once the reverse bias lowers, the junction breakdown is progressively reduced, thus possibly suppressing the flow of such carriers from the substrate to the SPAD junction.

\section{CONCLUSIONS}

We presented an extended experimental characterization of the memory effect occurring when a strong light pulse illuminates a silicon SPAD in time-gated acquisitions just before the gate-ON time interval of interest. The effect is similar to other background phenomena already reported in the literature, but, given the different structures and materials, they are probably due to very different processes. The origin of the memory effect is still not fully clear, but our measurements lead to the hypothesis that it takes place below the main depleted region, where usually photon absorption processes can be neglected.

Indeed, when a fast-gated SPAD acquisition setup is employed, the measurement dynamic range can reach $8 \mathrm{dec}-$ ades, thus allowing to detect such very faint amount of diffusing electrons that can cross the substrate depleted region and the highly doped buried layer.

In fact, this is what we discovered and investigated in this paper, where we have provided some further insight for the understanding of the ME phenomenon, and identified a first practical approach-tailor the substrate junction electric field and increase the buried-layer thickness - so as to reduce the ME. Even with present devices, a proper substrate bias can further increase the dynamic range by more than 4 orders of magnitude.

Focusing on the field of diffuse optics for non-invasive imaging and spectroscopy through biological tissues (see Refs. 1-5), the ME is actually the key limiting factor that prevents to increase the dynamic range beyond 8 decades. The chance to further improve on the suppression of early photons could open in the future exciting perspectives for real time imaging of brain function and diseases, ${ }^{33}$ and enable a new non-invasive optical modality in clinical diagnostics with high depth of view.

\section{ACKNOWLEDGMENTS}

The research leading to these results has received funding from the European Community 7th Framework Programme, under Grant Agreement No. 284464 (LASERLAB-EUROPE). 
${ }^{1}$ A. Pifferi, A. Torricelli, L. Spinelli, D. Contini, R. Cubeddu, F. Martelli, G. Zaccanti, A. Tosi, A. Dalla Mora, F. Zappa, and S. Cova, Phys. Rev. Lett. 100, 138101 (2008).

${ }^{2}$ A. Dalla Mora, A. Tosi, F. Zappa, S. Cova, D. Contini, A. Pifferi, L. Spinelli, A. Torricelli, and R. Cubeddu, IEEE J. Sel. Top. Quantum Electron. 16, 1023 (2010).

${ }^{3}$ E. Alerstam, T. Svensson, S. Andersson-Engels, L. Spinelli, D. Contini, A. Dalla Mora, A. Tosi, F. Zappa, and A. Pifferi, Opt. Lett. 37, 2877 (2012).

${ }^{4}$ M. Mazurenka, L. Di Sieno, G. Boso, D. Contini, A. Pifferi, A. Dalla Mora, A. Tosi, H. Wabnitz, and R. Macdonald, Biomed. Opt. Express 4, 2257 (2013).

${ }^{5}$ A. Puszka, L. Di Sieno, A. Dalla Mora, A. Pifferi, D. Contini, G. Boso, A. Tosi, L. Hervé, A. Planat-Chrétien, A. Koenig, and J. M. Dinten, Biomed. Opt. Express 4, 1351 (2013).

${ }^{6}$ W. Becker, A. Bergmann, M. A. Hink, K. Konig, K. Benndorf, and C. Biskup, Microsc. Res. Tech. 63, 58-66 (2004).

${ }^{7}$ G. Vicidomini, G. Moneron, K. Y. Han, V. Westphal, H. Ta, M. Reuss, J. Engelhardt, C. Eggeling, and S. W. Hell, Nat. Methods 8, 571-573 (2011). ${ }^{8}$ K. Vishwanath, B. Pogue, and M. A. Mycek, Phys. Med. Biol. 47, 3387-3405 (2002).

${ }^{9}$ A. Velten, T. Willwacher, O. Gupta, A. Veeraraghavan, M. G. Bawendi, and R. Raskar, Nat. Commun. 3, 745 (2012).

${ }^{10}$ G. Éthier-Majcher, P. St-Jean, G. Boso, A. Tosi, J. F. Klem, and S. Francoeur, Nat. Commun. 5, 3980 (2014).

${ }^{11}$ G. Boso, A. Dalla Mora, A. Della Frera, and A. Tosi, Sens. Actuators, A 191, 61 (2013).

${ }^{12}$ A. Tosi, A. Dalla Mora, F. Zappa, A. Gulinatti, D. Contini, A. Pifferi, L. Spinelli, A. Torricelli, and R. Cubeddu, Opt. Express 19, 10735 (2011).

${ }^{13}$ A. Dalla Mora, D. Contini, A. Pifferi, R. Cubeddu, A. Tosi, and F. Zappa, Appl. Phys. Lett. 100, 241111 (2012).

${ }^{14}$ S. M. Sze, Physics of Semiconductor Devices (Wiley, New York, 1981).
${ }^{15}$ S. Cova, A. Lacaita, and G. Ripamonti, IEEE Electron Device Lett. 12, 685 (1991).

${ }^{16}$ S. V. Polyakov and A. L. Migdall, Opt. Express 15, 1390 (2007).

${ }^{17}$ A. Migdall, S. Polyakov, J. Fan, and J. Bienfang, Single-Photon Generation and Detection (Academic Press, Waltham, 2013).

${ }^{18}$ F. Scholder, J.-D. Gautier, M. Wegmuller, and N. Gisin, Opt. Commun. 213, 57 (2002).

${ }^{19}$ M. Wegmuller, F. Scholder, and N. Gisin, J. Lightwave Technol. 22, 390 (2004).

${ }^{20}$ J. Zhang, R. Thew, J.-D. Gautier, N. Gisin, and H. Zbinden, IEEE J. Quantum Electron. 45, 792 (2009).

${ }^{21}$ P. Eraerds, M. Legré, J. Zhang, H. Zbinden, and N. Gisin, J. Lightwave Technol. 28, 952 (2010)

${ }^{22}$ G. Vincent, A. Chantre, and D. Bois, J. Appl. Phys. 50, 5484 (1979).

${ }^{23}$ T. Lunghi, E. Pomarico, C. Barreiro, D. Stucki, B. Sanguinetti, and H. Zbinden, Appl. Opt. 51, 8455 (2012).

${ }^{24}$ A. Gulinatti, P. Maccagnani, I. Rech, M. Ghioni, and S. Cova, Electron. Lett. 41, 272 (2005).

${ }^{25}$ M. Ghioni, A. Gulinatti, I. Rech, F. Zappa, and S. Cova, IEEE J. Sel. Top. Quantum Electron. 13, 852 (2007).

${ }^{26}$ P. B. Coates, J. Phys. E: Sci. Instrum. 1, 878 (1968).

${ }^{27}$ M. Rosling, H. Bleichner, P. Jonsson, and E. Nordlander, J. Appl. Phys. 76, 2855 (1994)

${ }^{28}$ A. Lacaita, M. Ghioni, and S. Cova, Electron. Lett. 25, 841 (1989).

${ }^{29}$ E. A. G. Webster, L. A. Grant, and R. K. Henderson, IEEE Trans. Electron Devices 60, 1188 (2013).

${ }^{30}$ W. Bludau, A. Onton, and W. Heinke, J. Appl. Phys. 45, 1846 (1974).

${ }^{31}$ A. Tosi, A. Dalla Mora, and F. Zappa, IEEE Photonics Technol. Lett. 20, $1956(2008)$

${ }^{32}$ R. Bube, Photoconductivity of Solids (Wiley, New York, 1960).

${ }^{33}$ A. Torricelli, D. Contini, A. Pifferi, M. Caffini, R. Re, L. Zucchelli, and L. Spinelli, NeuroImage 85, 28 (2014). 